\title{
Spectroscopic (FT-IR, Raman, NMR) and DFT Quantum Chemical Studies on Phenoxyacetic Acid and Its Sodium Salt
}

\author{
M. Samsonowicz, E. Regulska, and W. Lewandowski \\ Division of Chemistry, Bialystok University of Technology, 15-435 Bialystok, Poland
}

Correspondence should be addressed to E. Regulska, e.regulska@pb.edu.pl

Copyright (C) 2012 M. Samsonowicz et al. This is an open access article distributed under the Creative Commons Attribution License, which permits unrestricted use, distribution, and reproduction in any medium, provided the original work is properly cited.

\begin{abstract}
FT-IR, Raman, and NMR spectra of phenoxyacetic acid and its sodium salt were recorded and analyzed. Optimized geometrical structures of studied compounds were calculated by B3LYP/6-311++ $\mathrm{G}^{* *}$ method. The atomic charges were calculated by Mulliken, NPA (natural population analysis), APT (atomic polar tensor), MK (Merz-Singh-Kollman method), and ChelpG (charges from electrostatic potentials using grid-based method) methods. Geometric as well as magnetic aromaticity indices, dipole moments, and energies were also calculated. The theoretical wavenumbers and intensities of IR spectra as well as chemical shifts in ${ }^{1} \mathrm{H}$ and ${ }^{13} \mathrm{C}$ NMR spectra were obtained. The calculated parameters were compared with experimental characteristics of these molecules.
\end{abstract}

Keywords: Phenoxyacetic acid, sodium phenoxyacetate, FT-IR, Raman, DFT, molecular structure

\section{Introduction}

Phenoxyacetic acid has been investigated by various researches because of its biological activities. It is useful in the treatment of insulin resistance and hyperglycemia [1]. Derivatives of phenoxyacetic acid are widely used in herbicide and pesticide formulations. The molecular basis of their mode of action is not fully understood. The estimation of the electronic charge distribution in metal complexes and salts allows to predict what kind of deformation of the electronic system of ligand would undergo during complexation [2]. It also permits to make more precise interpretation of mechanism by which metals affect the biochemical properties of ligands. In this paper the influence of sodium cation on the electronic system of phenoxyacetic acid was studied.

\section{Experimental}

Sodium phenoxyacetate was prepared by dissolving the powder of phenoxyacetic acid in the water solution of the appropriate sodium hydroxide in a stoichiometric ratio $(1: 1)$. Both reagents were 
obtained from Aldrich Chemical Company. The solution was left at the room temperature for $24 \mathrm{~h}$ until the sample crystallized in the solid-state. Precipitants were filtered, washed by water, and dried under reduced pressure at $110^{\circ} \mathrm{C}$. Obtained complex was anhydrous-in the IR spectra of solid-state sample the lack of bands characterized for crystallizing water was observed.

The IR spectra were recorded with the Equinox 55, Bruker FT-IR spectrometer within the range $4000-400 \mathrm{~cm}^{-1}$. Samples in the solid state were measured in $\mathrm{KBr}$ pellets. The resolution of spectrometer was $1 \mathrm{~cm}^{-1}$. Raman spectra of solid samples in capillary tubes were recorded in the range of 4000$400 \mathrm{~cm}^{-1}$ with a FT-Raman accessory of the Perkin Elmer System 2000. The resolution of spectrometer was $1 \mathrm{~cm}^{-1}$. The NMR spectra of DMSO solution were recorded with the NMR AC $200 \mathrm{~F}$, Bruker unit. TMS was used as an internal reference.

The density functional (DFT) hybrid method B3LYP/6-311++G** was used to calculate optimized geometrical structures of studied compounds (Figure 1). All theoretical calculation were performed using the Gaussian'09 [3] of programs running on a PC computer.

\section{Results and Discussion}

\subsection{Vibrational Spectra}

Experimental and theoretical bands together with their relative intensities and band assignments for phenoxyacetic acid and its sodium salt in FT-IR and Raman spectra were obtained. Complete assignments of all bands require application of both IR and Raman methods supported by theoretical calculations and literature data [4]. The calculated wavenumbers were obtained by B3LYP method and 6$311++\mathrm{G}^{* *}$ basis set. The correlation between calculated and experimentally obtained wavenumbers in IR and Raman spectra of phenoxyacetic acid and sodium phenoxyacetate was studied and good agreement was found. The correlation coefficient $R^{2}$ for phenoxyacetic acid spectra is amount 0.9972 and for its sodium salt $R^{2}=0.9990$. The corresponding values for Raman spectra amount to 0.9969 and 0.9975 .

IR and Raman spectra for phenoxyacetic acid and its sodium salt are presented in Figure 1. Comparing results obtained for sodium phenoxyacetate to the, respectively, values of phenoxyacetic acid, certain changes of intensities and wavenumbers of the bands of aromatic system and carboxylic group can be noticed.

The changes of intensities and wavenumbers of the bands of aromatic system and carboxylate group in the case of sodium salt were discussed comparing to the free ligand. The characteristic bands occurring in the IR spectra of sodium phenoxyacetate, which do not exist in the spectra of free acid, for example: symmetric or asymmetric stretching vibrations $\nu(\mathrm{COO})$ and in plane $\beta(\mathrm{COO})$ and out of plane $\gamma(\mathrm{COO})$ deformations of carboxylic group were noticed. On the other hand, the lack of bands, which are characteristic for phenoxyacetic acid (the $\mathrm{C}=\mathrm{O}$ band, 1736 and $1703 \mathrm{~cm}^{-1} ; \beta(\mathrm{OH})$ band, $1300 \mathrm{~cm}^{-1}$, broad band of $\nu(\mathrm{OH})$, e.g.) were observed in the sodium salt spectra. The wavenumbers of aromatic bands numbered as 20b, 9b, 17a, and 5 in IR as well as in Raman spectra increase in comparison to free acid. For 2, 3, and $17 \mathrm{~b}$ bands the increase of wavenumbers was noticed only in IR spectra. In the case of $8 \mathrm{a}$ and $6 \mathrm{a}$ bands the decrease in comparison to phenoxyacetic acid was observed in IR spectra, whereas for 2, 3, 17b, and 19a bands in Raman the decrease of wavenumbers was noticed. There are also some changes of alky chain part of studied molecules. The wavenumbers of $\nu_{\mathrm{as}}\left(\mathrm{CH}_{2}\right)$ and $\nu\left(\mathrm{O}-\mathrm{CH}_{2}\right)$ bands shift to higher values in IR spectra of sodium phenoxyacetate, while $\nu_{s}\left(\mathrm{CH}_{2}\right)$ band shifts to lower values. 


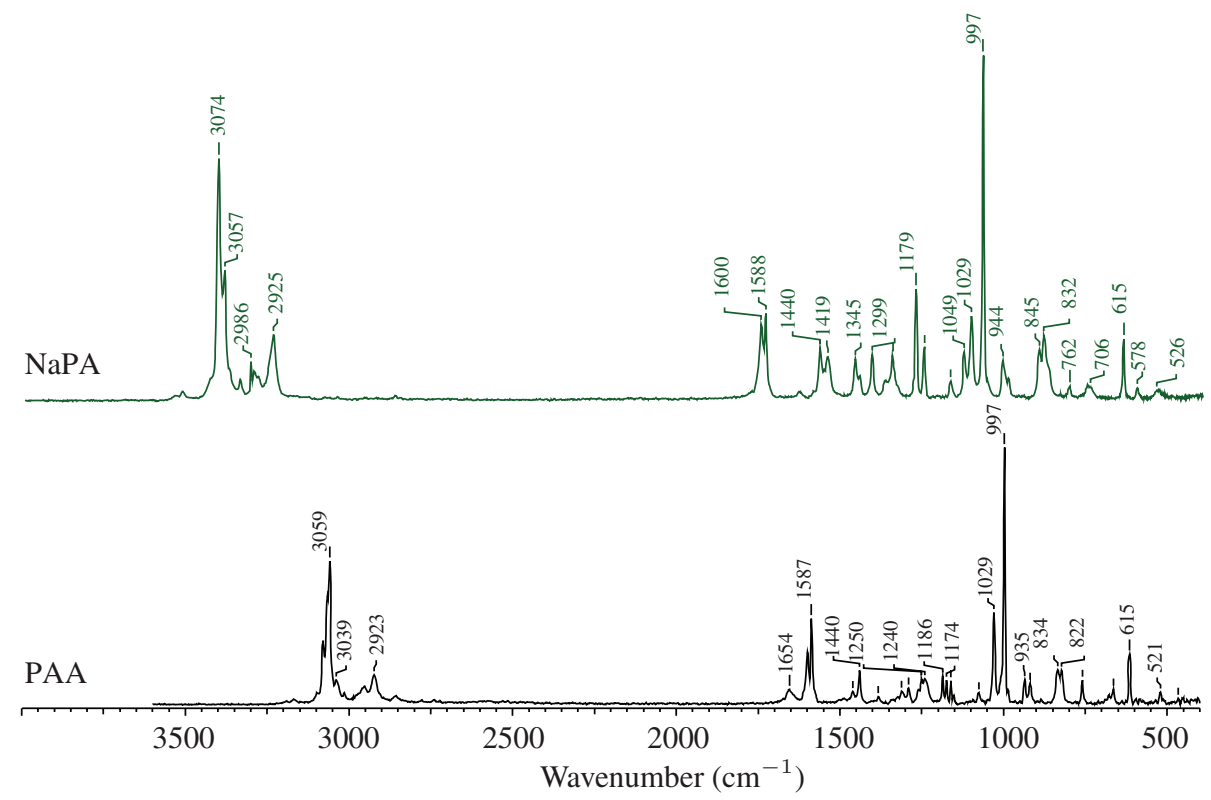

(a)
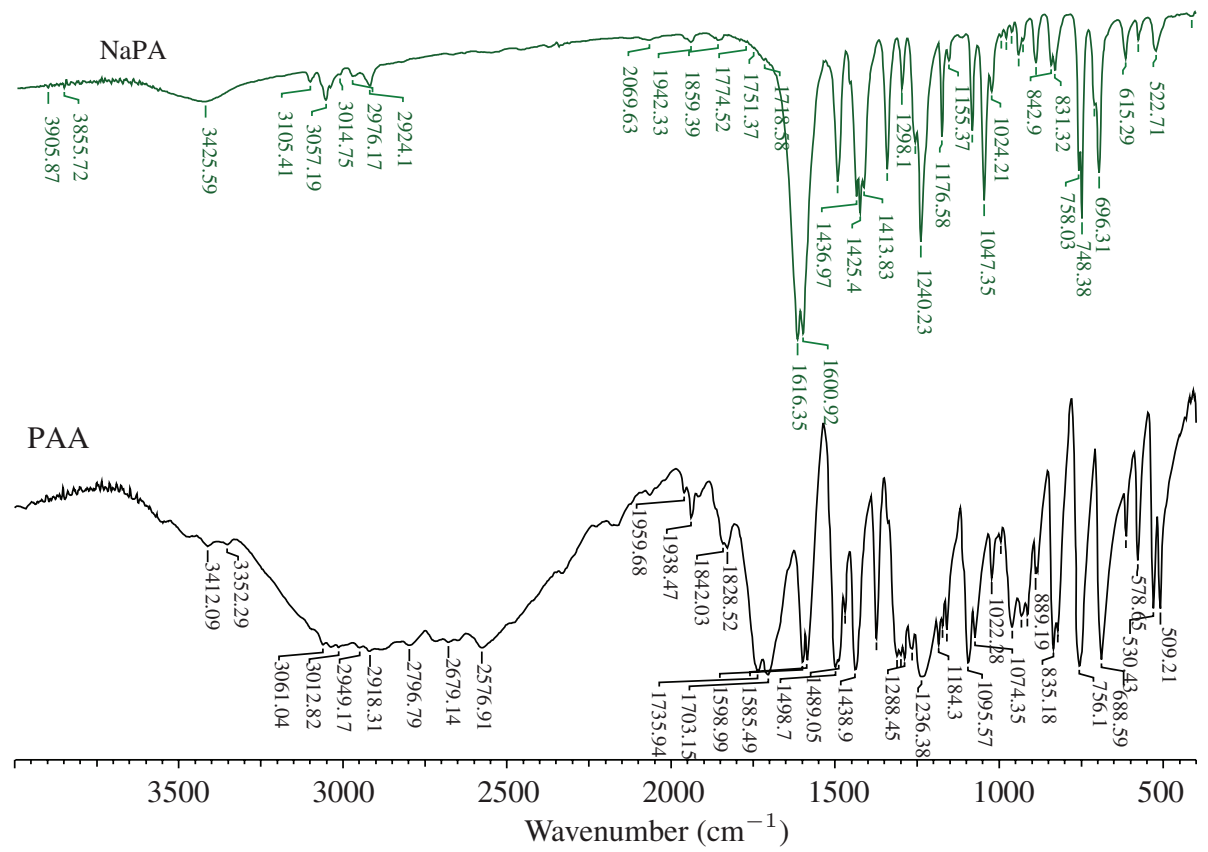

(b)

Figure 1: Raman (a) and IR (b) spectra for phenoxyacetic acid (PAA) and its sodium salt (NaPA). 


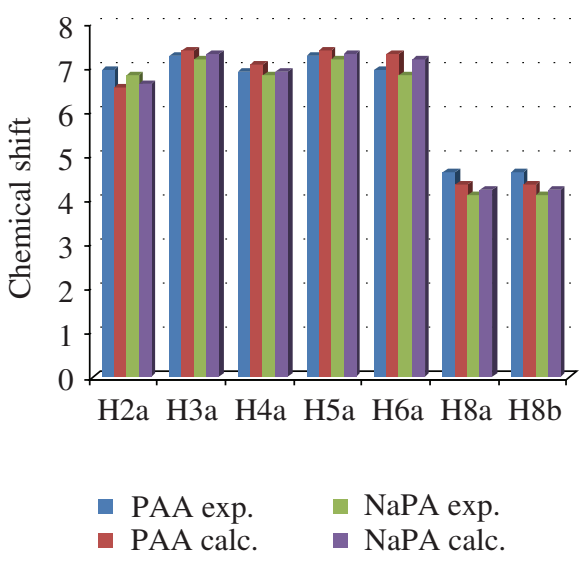

(a)

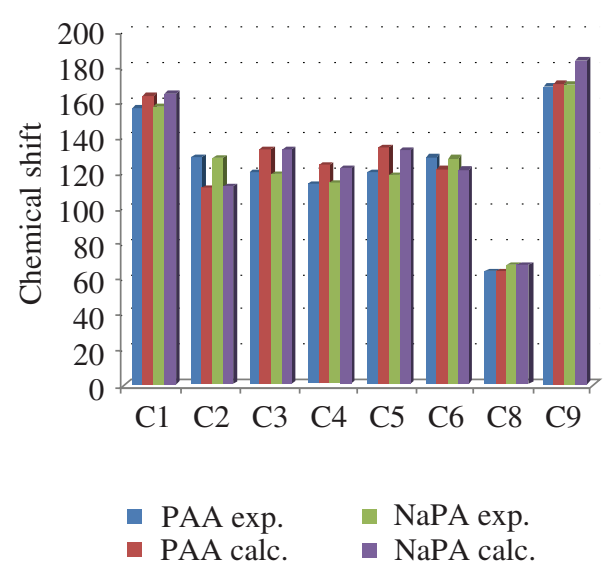

(b)

Figure 2: Calculated (B3LYP/6-311++G**) as well as experimental chemical shifts: (a) ${ }^{1} \mathrm{H}$ NMR, (b) ${ }^{13} \mathrm{C}$ NMR of phenoxyacetic acid (PAA) and its sodium salt (NaPA).

\subsection{NMR Spectra}

Theoretically as well as experimentally obtained ${ }^{1} \mathrm{H}$ NMR and ${ }^{13} \mathrm{C}$ NMR chemical shifts of phenoxyacetic acid and its sodium salt are presented in Figure 2. The linear correlation between proton as well as carbon NMR shieldings of studied compounds and experimental data is observed. The correlation coefficient $\left(R^{2}\right)$ for ${ }^{1} \mathrm{H}$ NMR spectra are amount to 0.9739 for phenoxyacetic acid (PAA) and 0.9868 for sodium phenoxyacetate (NaPA). For ${ }^{13} \mathrm{C}$ NMR spectra corresponding values are 0.8945 and 0.9035 . All protons in sodium phenoxyacetate are shifted diamagnetically in comparison to PAA. This tendency suggests that introduction of sodium atom causes the decrease in ring current intensity. Some changes in ${ }^{13} \mathrm{C}$ NMR spectra were also observed. The chemical shifts of almost all carbon atoms in sodium phenoxyacetate molecule, except of $\mathrm{C} 1, \mathrm{C} 8$, and $\mathrm{C} 9$ atoms, are lower than those in free acid.

\subsection{Calculated Molecular Structure}

Optimized geometrical structures of phenoxyacetic acid as well as of sodium phenoxyacetate molecule were obtained using B3LYP/6-311++ $\mathrm{G}^{* *}$ method. The bond lengths and the angles between bonds in sodium salt molecule in comparison to free acid were presented in Table 1 . The increase of almost all bond lengths in aromatic ring except of $\mathrm{C} 2-\mathrm{C} 3$ and $\mathrm{C} 5-\mathrm{C} 6$ bonds in sodium salt molecule in comparison to acid was observed. The increase of $\mathrm{O} 7-\mathrm{C} 8, \mathrm{C} 8-\mathrm{C} 9, \mathrm{C} 9-\mathrm{O} 10$, and $\mathrm{O} 10-11$ a bond lengths was also noticed, whereas bond lengths of $\mathrm{C} 1-\mathrm{O} 7, \mathrm{C} 9-\mathrm{O} 11$, and O11-11a decreased in NaPA in comparison to PAA molecule. The differences between C9-O10 and C9-O11 as well as O10-11a and O11-11a bond lengths almost disappeared. In the case of angles, the increase was observed for almost all angles in the aromatic ring, except of $\mathrm{C} 3-\mathrm{C} 4-\mathrm{C} 5$ and $\mathrm{C} 6-\mathrm{C} 1-\mathrm{C} 2$ angles in NPA molecule. For C2-C1-O7 and O7C8-C9 angles the increase was observed, but decrease was noticed only for C8-C9-010, C9-O11-11a, and C9-O10-11a angles. 
Table 1: The bond lengths, angles, aromatic indices, values of dipole moment, and energy calculated (B3LYP/6-311++G**) for alkali metal phenoxyacetates.

\begin{tabular}{|c|c|c|c|}
\hline Atoms & PAA & $\mathrm{NaPA}$ & $\begin{array}{l}\text { Atomic numbers of PAA and } \\
\text { NaPA molecules }\end{array}$ \\
\hline \multicolumn{4}{|c|}{ Bond lengths $(\AA)^{\mathrm{a}}$} \\
\hline $\mathrm{C} 1-\mathrm{C} 2$ & 1.3963 & 1.3992 & \\
\hline $\mathrm{C} 2-\mathrm{C} 3$ & 1.3981 & 1.3977 & \\
\hline C3-C4 & 1.3897 & 1.3901 & \\
\hline $\mathrm{C} 4-\mathrm{C} 5$ & 1.3980 & 1.3984 & \\
\hline C5-C6 & 1.3877 & 1.3873 & \\
\hline $\mathrm{C} 6-\mathrm{C} 1$ & 1.3995 & 1.4024 & \\
\hline $\mathrm{C} 1-\mathrm{O} 7$ & 1.3706 & 1.3617 & \\
\hline $\mathrm{O} 7-\mathrm{C} 8$ & 1.4038 & 1.4162 & \\
\hline C8-C9 & 1.5174 & 1.5290 & \\
\hline C9-O10 & 1.1977 & 1.2559 & \\
\hline C9-O11 & 1.3576 & 1.2689 & (3) \\
\hline O10-H(Na)11a & 0.9689 & 2.2169 & \\
\hline $\mathrm{O} 11-\mathrm{H}(\mathrm{Na}) 11 \mathrm{a}$ & 2.3148 & 2.2199 & \\
\hline \multicolumn{4}{|c|}{ Angles $\left(^{\circ}\right)$} \\
\hline $\mathrm{C} 1-\mathrm{C} 2-\mathrm{C} 3$ & 119.34 & 119.55 & \\
\hline $\mathrm{C} 2-\mathrm{C} 3-\mathrm{C} 4$ & 120.89 & 121.01 & \\
\hline $\mathrm{C} 3-\mathrm{C} 4-\mathrm{C} 5$ & 119.24 & 119.07 & \\
\hline C4-C5-C6 & 120.61 & 120.63 & \\
\hline C5-C6-C1 & 119.84 & 120.14 & \\
\hline $\mathrm{C} 6-\mathrm{C} 1-\mathrm{C} 2$ & 120.10 & 119.59 & \\
\hline $\mathrm{C} 6-\mathrm{C} 1-\mathrm{O} 7$ & 115.28 & 115.55 & \\
\hline $\mathrm{C} 2-\mathrm{C} 1-\mathrm{O} 7$ & 124.62 & 124.87 & \\
\hline $\mathrm{C} 1-\mathrm{O} 7-\mathrm{C} 8$ & 118.49 & 118.64 & \\
\hline O7-C8-C9 & 108.85 & 111.45 & \\
\hline C8-C9-O10 & 127.23 & 120.84 & \\
\hline C8-C9-O11 & 109.00 & 114.37 & \\
\hline C9-O11-H(Na)11a & 107.43 & 87.23 & \\
\hline O10-C9-O11 & 123.78 & 124.79 & \\
\hline $\mathrm{H}(\mathrm{Na}) 11 \mathrm{a}-\mathrm{O} 10-\mathrm{C} 9$ & 107.43 & 87.41 & \\
\hline \multicolumn{4}{|c|}{ Geometric aromaticity indices } \\
\hline $\mathrm{HOMA}^{\mathrm{b}}$ & 0.982557 & 0.976809 & \\
\hline$A_{\mathrm{J}}^{\mathrm{c}}$ & 0.99765 & 0.99672 & Hy \\
\hline $\mathrm{BAC}^{\mathrm{d}}$ & 0.93095 & 0.92338 & \\
\hline$I_{6}{ }^{\mathrm{e}}$ & 96.21196 & 95.52024 & \\
\hline \multicolumn{4}{|c|}{ Magnetic aromaticity indices } \\
\hline $\mathrm{NICS}^{\mathrm{f}}$ & -11.0192 & -8.8957 & \\
\hline Dipole moment $(D)$ & 2.48 & 6.99 & \\
\hline Energy (hartree) $)^{g}$ & -535.5 & -542.55 & \\
\hline
\end{tabular}

${ }^{\mathrm{a}} 1 \AA=10^{-10} \mathrm{~m} ;{ }^{\mathrm{b}}$ abbreviation from Harmonic Oscillator Model of Aromaticity; ${ }^{\mathrm{c}}$ normalized function of variance of bond lengths; ${ }^{\mathrm{d}}$ bond alternation coefficient; ${ }^{\mathrm{e}}$ Bird's index [5]; ${ }^{\mathrm{f}}$ nuclear independent chemical shifts; ${ }^{\mathrm{g}} 1$ hartree $=2625.5 \mathrm{~kJ} / \mathrm{mol}$. 


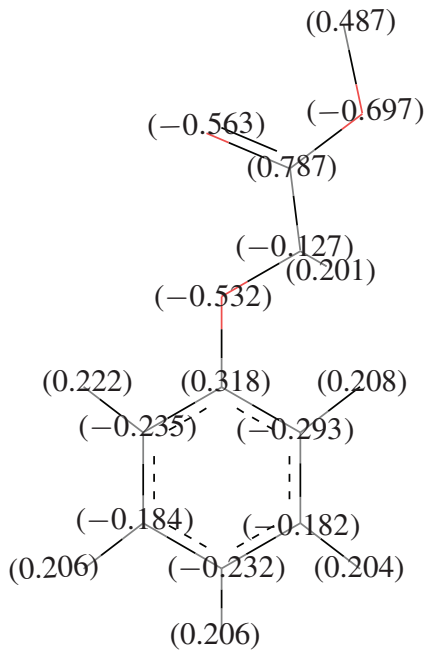

(a)
(0.919)

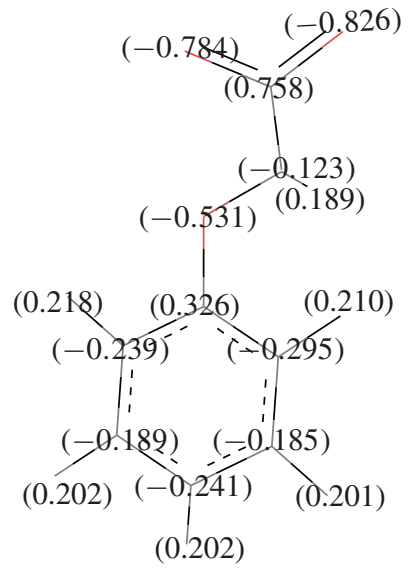

(b)

Figure 3: Electronic charge distribution (NPA method) calculated for molecules of phenoxyacetic acid (a) and its sodium salt (b).

Geometric and magnetic aromaticity indices [5-7], dipole moments, and energies were calculated and also shown in Table 1. All geometric aromaticity indices calculated for sodium phenoxyacetate in comparison to acid molecule decreased. It indicates that aromaticity of salt molecule decreased in comparison to free acid. This conclusion was confirmed by values of magnetic aromaticity indices NICS.

Mulliken, NPA, APT, MK, and ChelpG methods were used to calculate atomic charges on the atoms of phenoxyacetic acid molecule and its sodium salt. One of them, exemplary, is presented in Figure 3. The highest changes irrespective of used method are observed for carboxylate group. Total charges calculated for $\mathrm{COO}$ significantly decrease in comparison to free acid, for example, the values calculated by the Mulliken method for PAA amount to -0.766 , but for NaPA molecule -1.194 . In the case of other methods corresponding values are -0.473 and -0.852 (NPA); -0.292 and -0.763 (APT); -0.473 and $0.835(\mathrm{MK}) ;-0.341$ and -0.851 (ChelpG method).

\section{Conclusions}

Replacement of hydrogen by sodium in molecule causes significant changes in geometrical structure of studied molecules. The highest changes are noticed for carboxylate group, as may be expected. However almost all bond lengths in aromatic ring insignificantly increase, the decrease of aromaticity of studied molecules are observed. The displacements of bands in FT-IR, Raman, as well as in NMR spectra are also noticed. In IR and Raman spectra, different changes of bands are observed, some of them shift to higher, other to lower, wavenumbers. In ${ }^{1} \mathrm{H}$ and ${ }^{13} \mathrm{C}$ NMR spectra almost all bands shift to lower values in sodium phenoxyacetate spectra in comparison to free acid. It is characteristic tendency for molecules, in which the decrease of aromaticity is noticed. 


\section{Acknowledgment}

Presented work was supported by Białystok University of Technology (Theme no. N N312 427639).

\section{References}

[1] N. Sundaraganesan, C. Meganathan, B. Anand, and C. Lapouge, "FT-IR, FT-Raman spectra and ab initio DFT vibrational analysis of p-bromophenoxyacetic acid," Spectrochimica Acta Part A, vol. 66, no. 3, pp. 773-780, 2007.

[2] W. Lewandowski, M. Kalinowska, and H. Lewandowska, "The influence of metals on the electronic system of biologically important ligands. Spectroscopic study of benzoates, salicylates, nicotinates and isoorotates," Journal of Inorganic Biochemistry, vol. 99, no. 7, pp. 1407-1423, 2005.

[3] M. J. Frisch, G. W. Trucks, H. B. Schlegel et al., Gaussian 09 (Revision A.1), Gaussian, Inc., Wallingford, Conn, USA, 2009.

[4] G. Varsányi, Assignments for Vibrational Spectra of 700 Benzene Derivatives, Akademiai Kiado, Budapest, Hungary, 1973.

[5] T. M. Krygowski and M. Cyrański, "Separation of the energetic and geometric contributions to the aromaticity of $\pi$-electron carbocyclics," Tetrahedron, vol. 52, no. 5, pp. 1713-1722, 1996.

[6] E. Regulska, M. Samsonowicz, R. Świstocka, and W. Lewandowski, "Molecular structure of alkali metal 4-nitrobenzoates," Journal of Physical Organic Chemistry, vol. 20, no. 2, pp. 93-108, 2007.

[7] P. Von Ragué Schleyer, M. Manoharan, Z. X. Wang et al., "Dissected nucleus-independent chemical shift analysis of $\pi$-aromaticity and antiaromaticity," Organic Letters, vol. 3, no. 16, pp. 2465-2468, 2001 . 


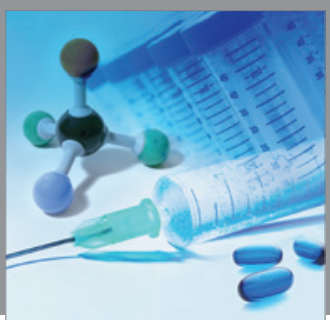

International Journal of

Medicinal Chemistry

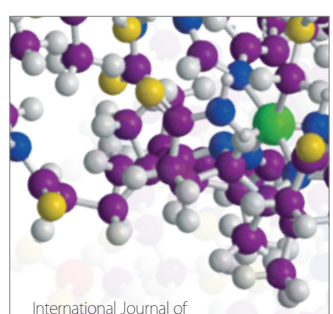

Carbohydrate Chemistry

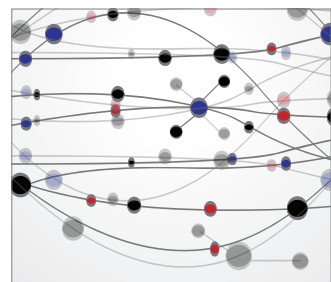

The Scientific World Journal
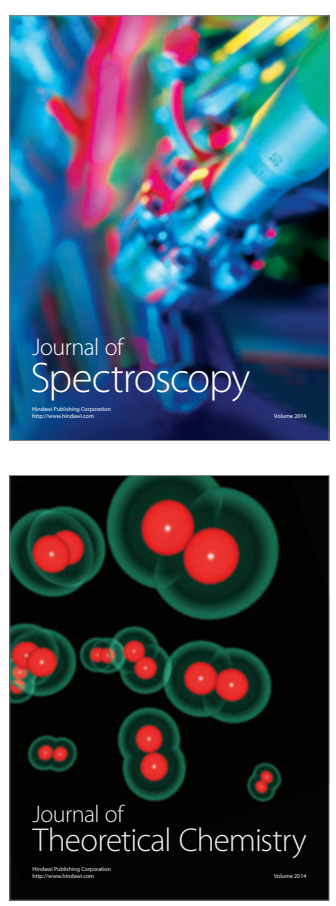
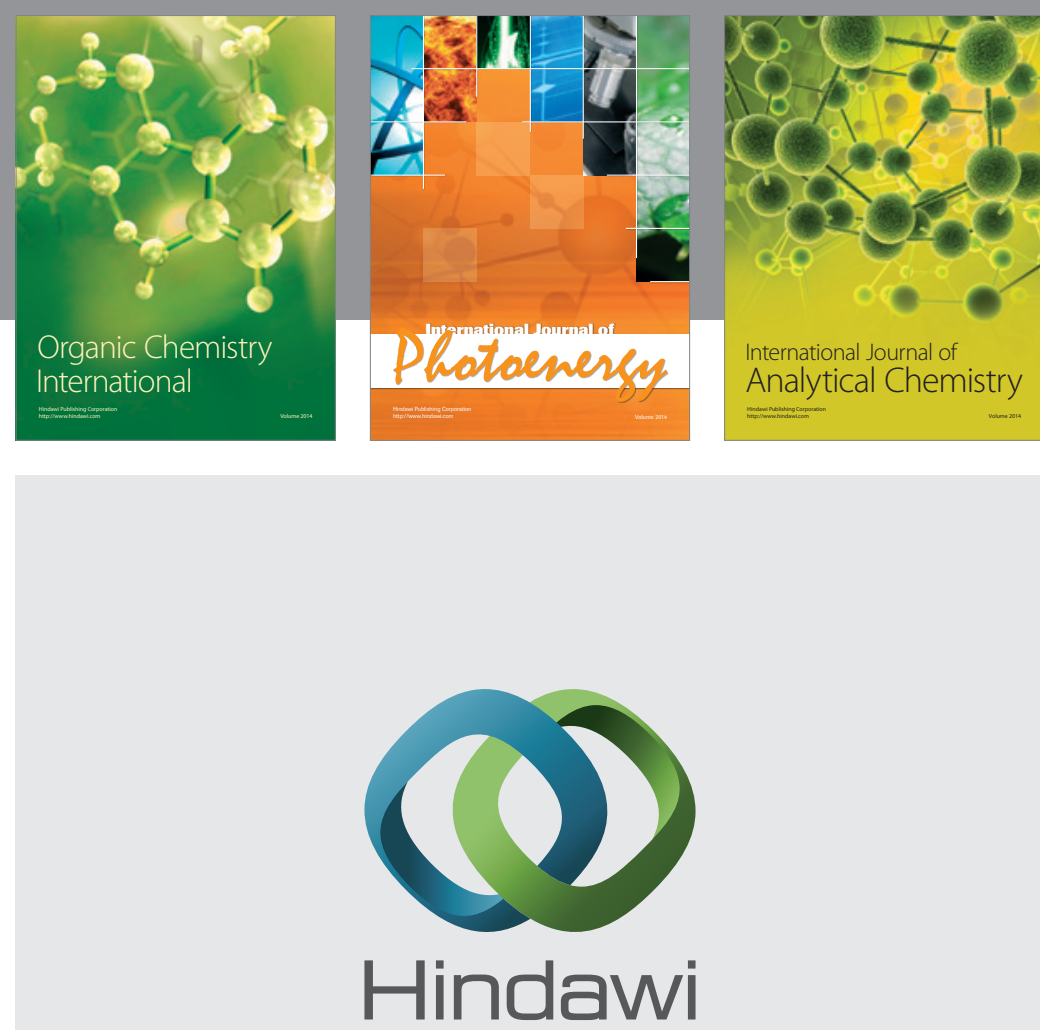

Submit your manuscripts at

http://www.hindawi.com
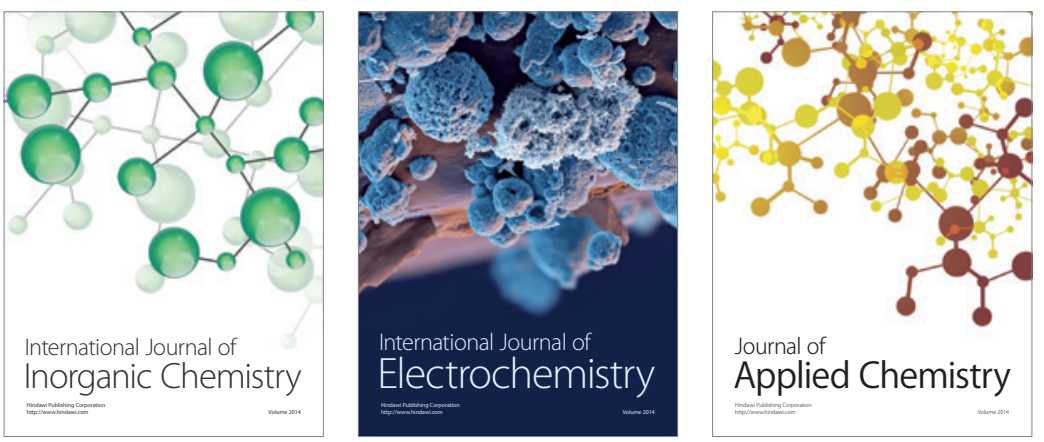

Journal of

Applied Chemistry
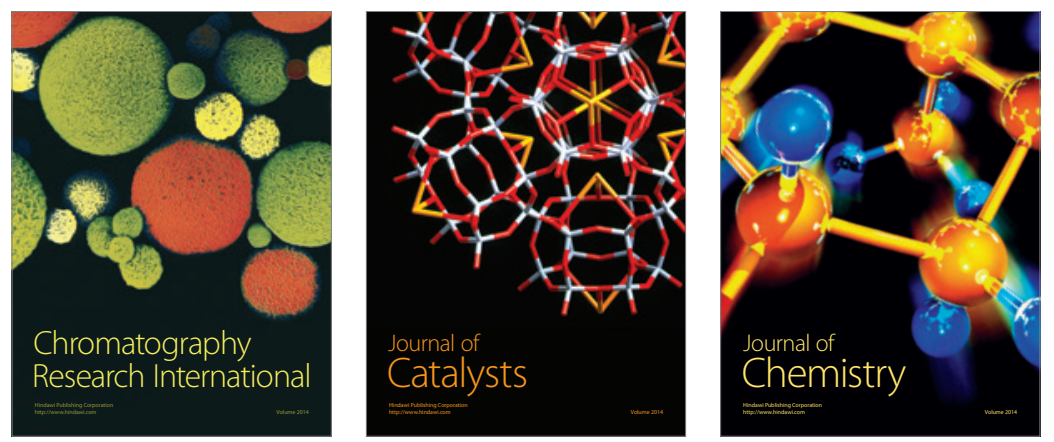
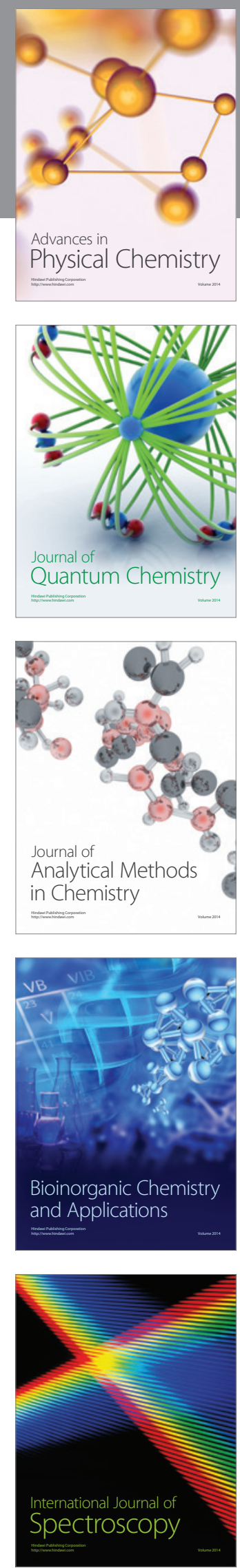